INRA Prod. Anim., 2008, 21 (1), 5-10

\title{
La revue INRA Productions Animales a 20 ans
}

J.-M. PEREZI, M.-H. FARCE ${ }^{2}$, D. CASTE 3

1 INRA, Département Physiologie Animale et Systèmes d'Elevage, F-31326 Castanet-Tolosan, France 2 INRA, SupAgro, UMR868 Elevage des Ruminants en Régions Chaudes, F-34060 Montpellier, France

${ }^{3}$ INRA, UE333, Domaine de la Verrerie, F-81400 Blaye-les-Mines, France

Courriel : Jean-Marc.Perez@toulouse.inra.fr

\section{1 / Un peu d'histoire...}

C'est en juin 1984, au cours d'une réunion du Bureau des Productions Animales de l'INRA consacrée aux publications, qu'est née l'idée d'une revue de transfert dans le domaine animal. Extrait du compte-rendu (18/06/84) : «Il a été décidé d'étudier la création d'une revue des Productions Animales par élargissement du Bulletin de Theix [...]. Un comité est chargé de mettre l'opération en place, sous la responsabilité de C. MALTERRE (Elevage des Herbivores, Theix), avec des représentants des divers secteurs et centres concernés: B. SAUVEUR (Aviculture, Tours), J.-M. PEREZ (Recherche Porcine, Rennes), B. CHEVASSUS (Hydrobiologie, Jouy), R. FENSTERBANK (Pathologie, Tours)».

De 1970 à 1987, le «Bulletin technique du CRZV de Theix » avait permis de faire connaître à un large public, essentiellement sous la forme de résultats expérimentaux, les travaux conduits sur les ruminants par les chercheurs de ce centre de recherches. La nécessité de faire évoluer la formule provenait du constat suivant : d'une part la contribution des chercheurs INRA extérieurs au centre de Clermont-Ferrand/Theix avait fortement augmenté au début des années 80 , d'autre part les lecteurs du Bulletin souhaitaient trouver des synthèses sur les travaux de l'ensemble du secteur des productions animales de l'INRA, sous une forme plus accessible. Il a donc été décidé : 1 / d'étendre les sujets traités à toutes les espèces animales d'intérêt zootechnique et à d'autres thématiques scientifiques que celles abordées à Theix ; $2 /$ de privilégier la publication d'articles de synthèse.

Il a fallu du temps et une bonne dose de persévérance pour que, ouverture de poste et de crédits aidant, se concrétise cette idée de création d'une revue de synthèse avec le label INRA dans le secteur animal. En janvier 1987, M.-H. FARCE était recrutée pour assurer la responsabilité de la rédaction, responsabilité qu'elle a assumée jusqu'en 2004. Le comité opérationnel, précédemment évoqué, s'est élargi rapidement pour constituer un véritable comité de rédaction pluridisciplinaire avec le souci de représenter les différentes espèces animales et pour assurer également une bonne couverture géographique des centres INRA concernés par les recherches dans le domaine animal.

En février 1988, sortait enfin le premier numéro de la revue INRA Productions Animales.

Au fil des années, les responsables ont changé. En 1990, le tandem MALTERRE/SAUVEUR faisait place au trio Demarquilly/Micol/SAuveur. En février 1999, la Direction Scientifique du secteur des «Productions Animales» de l'INRA a demandé à l'un d'entre nous (J.-M. PEREZ) d'assumer la responsabilité de la revue et de renouveler son comité de rédaction. En novembre 2004, après le départ de M.-H. FARCE, ce dernier prenait également en charge la Rédaction en faisant équipe avec D. CASTE pour la partie édition.

A partir de cette date, c'est l'ensemble de la chaîne de publication de la revue (rédaction/édition/fabrication/ impression) qui se trouvait ainsi localisée à Toulouse ; seule l'activité de gestion des abonnements continuant d'être assurée à Versailles par le Service des Editions de l'INRA, désormais regroupé au sein des Editions Quae avec ses homologues du Cemagref, du Cirad et de l'Ifremer.

Parallèlement aux changements de responsable, le comité de rédaction a évolué dans sa composition et comprend actuellement 12 chercheurs. $\mathrm{Au}$ total, plus de 50 personnes ont participé à cette aventure collective entre 1988 et 2008 (encadré 1).

\begin{tabular}{|c|c|c|c|}
\hline \multicolumn{4}{|c|}{ Une œuvre collective } \\
\hline $\begin{array}{l}\text { G. Aumont } \\
\text { G. Baril }{ }^{(2)} \\
\text { P. Berge } \\
\text { M. Bonneau } \\
{ }^{(1)} \\
\text { C. Boulard } \\
\text { G. Brulé } \\
\text { J. Cabaret } \\
\text { D. Caste } \\
\text { B. Charley } \\
\text { P. Chemineau } \\
\text { M. Chenost } \\
\text { (1) } \\
\text { B. Chevassus } \\
\text { N. Chonion }\end{array}$ & $\begin{array}{l}\text { G. Choubert } \\
\text { Y. De Fontaubert } \\
\text { L. Delaby }{ }^{(2)} \\
\text { C. Demarquilly } \\
\text { J.-Y. Dourmad } \\
\text { B. Dumont }{ }^{(2)} \\
\text { J.-M. Elsen } \\
\text { M.-H. Farce } \\
{ }^{(1)} \\
\text { F. Grosclaude } \\
\text { L. Gruner } \\
\text { L. Gueguen }{ }^{(1)} \\
\text { J.-F. Guillot } \\
\text { P. Herpin }\end{array}$ & $\begin{array}{l}\text { A. Hoden } \\
\text { J. Hommel }^{(1)} \\
\text { F. Lebas } \\
{ }^{(1)} \\
\text { B. Leclercq } \\
\text { J. Le Dividich }^{(1)} \\
\text { N. Le Floc'h }{ }^{(2)} \\
\text { G. Maisse } \\
\text { C. Malterre } \\
\text { C. } \\
\text { M. Mambrini } \\
\text { J.-P. Melcion } \\
\text { F. Meschy } \\
\text { D. Micol } \\
\text { G. Monin }\end{array}$ & $\begin{array}{l}\text { Y. Nys }{ }^{(2)} \\
\text { J.-M. Perez }^{(1)(2)} \\
\text { M. Petit }^{(2)} \\
\text { B. Picard } \\
\text { M. Picard } \\
\text { G. Renand }{ }^{(2)} \\
\text { E. Rocher } \\
\text { M. Roux }{ }^{(1)} \\
\text { D. Sauvant } \\
{ }^{(2)} \\
\text { B. Sauveur }{ }^{(1)} \\
\text { P. Sellier }{ }^{(1)} \\
\text { J.-L. Troccon } \\
\text { P. Volland-Nail }\end{array}$ \\
\hline $\begin{array}{l}\text { - }{ }^{(1)} 1^{\mathrm{er}} \text { Comité de } \\
\text { - }{ }^{(2)} \text { Comité de réd } \\
\text { - en gras : les dire } \\
\text { - soulignés : les re }\end{array}$ & $\begin{array}{l}\text { Ion (février 1988) } \\
\text { actuel (février 2008) } \\
\text { scientifiques succes } \\
\text { ables successifs de }\end{array}$ & $\begin{array}{l}\text { e la revue } \\
\text { action et de l'édition }\end{array}$ & \\
\hline
\end{tabular}




\section{2 / Objectif et fonctionne- ment de la revue}

Malgré les changements successifs de responsables, l'objectif de la revue n'a pas varié. Depuis l'origine, il s'agit :

- de publier des articles de synthèse sur tous les sujets concernant les productions animales,

- à destination d'un public francophone d'utilisateurs des résultats de la recherche,

- pour mieux faire connaître les travaux de l'INRA.

Le fonctionnement de cette revue à vocation de synthèse ne diffère pas fondamentalement de celui des revues scientifiques diffusant des résultats de recherche «primaires», avec en particulier l'application de la même règle de soumission de chaque article à l'analyse critique de deux chercheurs avant sa publication.

Mais, l'objectif de diffusion auprès d'un public assez large nécessite en outre une rédaction dans un style plus abordable que celui des articles scientifiques destinés à des chercheurs spécialisés, associée à une présentation moins classique (avec un chapeau introductif, des encadrés, des illustrations plus nombreuses...). Cela se traduit aussi par un travail important, souvent méconnu et sous-estimé, de remise en forme voire de réécriture des textes par la Rédaction de la revue pour en améliorer la lisibilité pour des lecteurs non-spécialistes.

Autre différence notable par rapport à une revue de type «primaire» : les articles n'arrivent pas spontanément. Il faut suggérer la rédaction de mises au point sur des sujets d'actualité, proposer des numéros à thème, solliciter en permanence les chercheurs pour qu'ils valorisent leurs travaux sous forme d'articles de synthèse, etc. Soulignons à cet égard le rôle important des membres du comité de rédaction qui effectuent un travail patient de prospection auprès des chercheurs dans leurs domaines de prédilection respectifs.

\section{3 / Evolution éditoriale}

Le rythme de parution a été fixé à l'origine à 5 numéros par an. Depuis 1988, la revue a évolué (cf. encadré 2) notamment avec la publication de

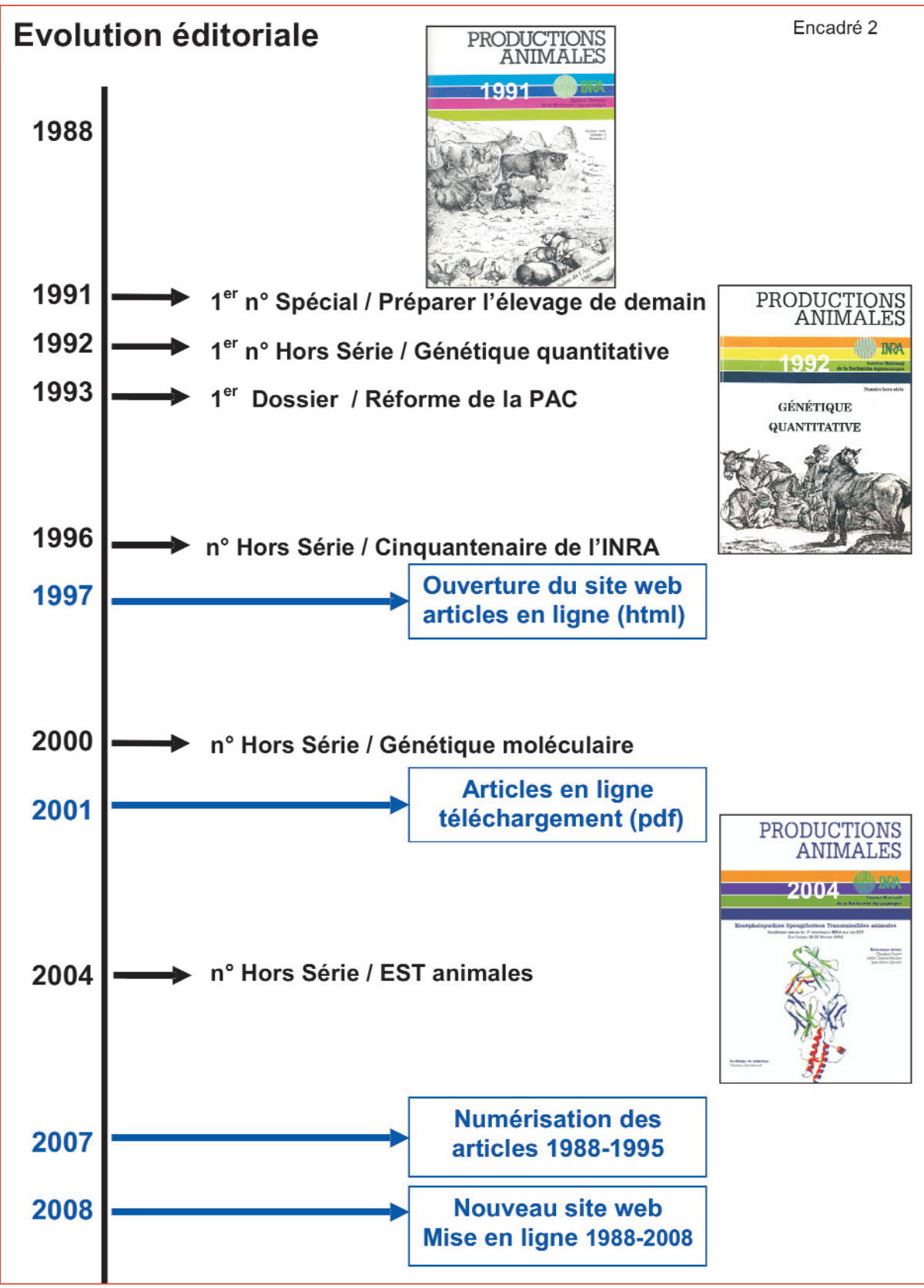

numéros spéciaux et de dossiers thématiques comportant plusieurs textes sur un même sujet d'actualité :

- le premier numéro spécial, consacré à «L'élevage de demain», est sorti en 1991 à l'occasion du Salon de l'Agriculture. En raison du succès de la formule, ces numéros à thème ont été publiés à partir de 1996 au rythme régulier d'au moins un par an. Au total, 15 numéros spéciaux ont été ainsi édités entre 1988 et 2008 (encadré 3).

- le premier «dossier» est sorti en 1993. Il était consacré à la réforme de la PAC. Au total, 12 dossiers de ce type ont été publiés. Parmi les plus récents, citons notamment un dossier consacré à l'alimentation des ruminants à l'occasion de la sortie du nouveau «Livre rouge» (encadré 4$)$.
La revue a également publié quatre numéros hors série (c.-à-d. hors abonnement) pour présenter un bilan complet des travaux dans certaines disciplines scientifiques ou sur une question d'actualité :

- 1992 : Génétique quantitative (B. Bibé et al), 302p.

- 1996: Cinquante ans de recherches en productions animales (F. Grosclaude, Y. Geay, M.-H. Farce), numéro édité à l'occasion du cinquantenaire de l'INRA, 152p.

- 2000 : Génétique moléculaire (B. Bibé, P. Mulsant, P. Sellier), 262p.

- 2004 : Encéphalopathies Spongiformes Transmissibles animales (J.-M. Elsen, C. Ducrot, J. Charley-Poulain, J.-M. Aynaud), 131p. 


\section{4 / Contenu des articles}

\section{1 / Répartition des articles par espèce animale cible}

Un tiers des articles parus concernent les ruminants (bovins, ovins, caprins), $1 / 3$ les animaux monogastriques (porc, lapin, volailles), le cheval et les poissons, et $1 / 3$ traitent de sujets généraux pluri-espèces (figure 1).

Même s'ils sont peu nombreux, les articles relatifs aux espèces de moindre importance économique ont souvent un très grand succès si l'on en juge par le nombre très élevé de téléchargements des tirés à part. Ainsi par exemple, un article sur 1'escargot publié en 1996 a été téléchargé plus de 18000 fois en 6 ans !

\section{2 / Répartition des articles par thématique de recherches}

Plus de la moitié des articles publiés concernent la physiologie des animaux (nutrition, reproduction, comportement...) et les méthodes d'élevage, $20 \%$ la génétique (mais essentiellement via les numéros hors série) et $4 \%$ la santé animale, comme le montre la répartition des articles par département de recherche INRA d'origine du premier auteur (tableau 1)

Si les 3/4 des articles concernent directement la biologie des animaux et leur élevage, la revue publie aussi des articles relatifs au développement agricole et à l'économie des filières.

\section{Les numéros spéciaux}

Encadré 3

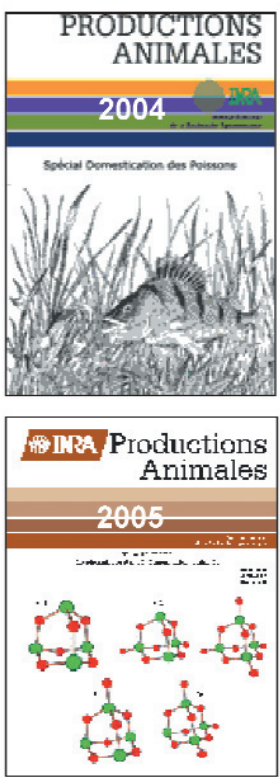

1991 Préparer l'élevage de demain

1996 Photopériode et reproduction

1997 Elevage des petits ruminants en zone tropicale

1998 Biotechnologies animales

1999 Lipogenèse et qualité des produits animaux

2000 Granulométrie des aliments et nutrition animale

2001 Matières grasses alimentaires et qualité des produits animaux

2003 Quel avenir pour les filières animales?

2004 Domestication des poissons

2005 Le phosphore dans l'alimentation animale

2006 Mitochondries et biologie du muscle

Hormones et promoteurs de croissance

2007 Bien-être animal

Santé des espèces aquacoles

200820 ans de recherches en productions animales à l'INRA

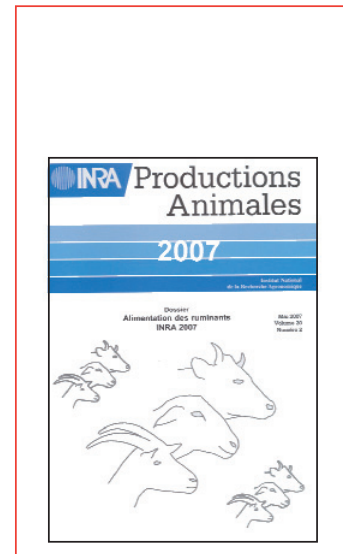

\section{Les dossiers thématiques}

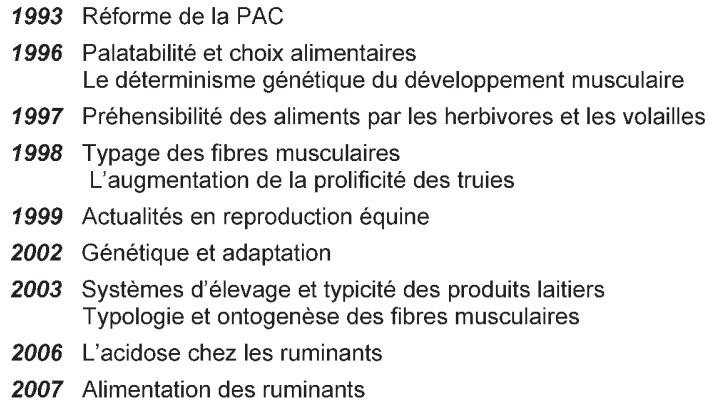

Figure 1. Répartition des articles publiés (1988-2007) par Tableau 1. Répartition des articles publiés (1988-2007) par départeespèce animale cible.

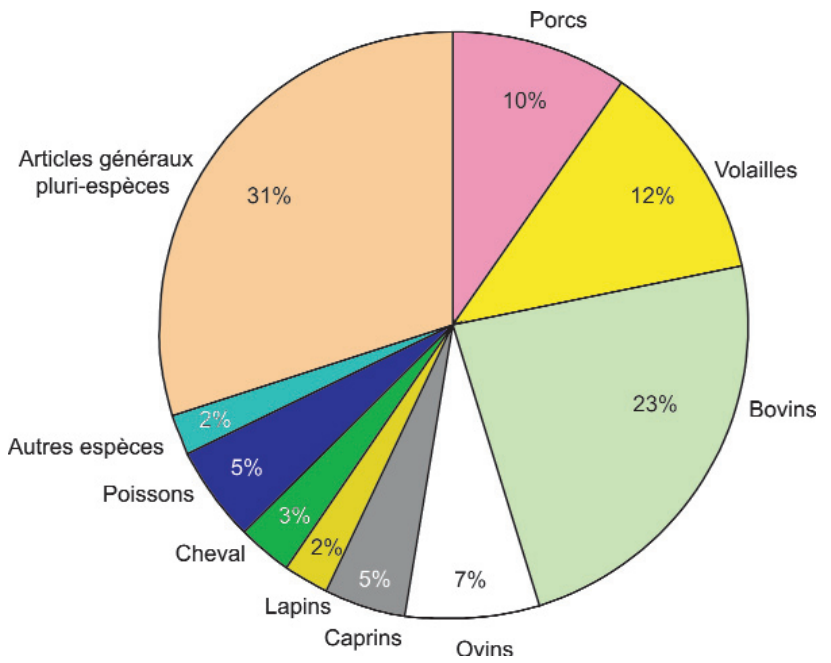
ment de recherche INRA ${ }^{1}$.

\begin{tabular}{|l|c|c|c|}
\hline \multirow{2}{*}{\multicolumn{1}{|c|}{ Départements }} & \multicolumn{2}{c|}{ Articles dans } & \multirow{2}{*}{ Total } \\
\cline { 2 - 3 } & $\mathbf{n}^{\circ}$ courants & $\mathbf{n}^{\circ}$ hors série & \\
\hline $\begin{array}{l}\text { Physiologie Animale } \\
\text { et Systèmes d'Elevage }\end{array}$ & 411 & 9 & 420 \\
\hline Génétique Animale & 63 & 97 & 160 \\
\hline Santé Animale & 21 & 8 & 29 \\
\hline $\begin{array}{l}\text { Economie } \\
\text { et Sociologie Rurales }\end{array}$ & 44 & & 44 \\
\hline $\begin{array}{l}\text { Sciences pour I'Action } \\
\text { et le Développement }\end{array}$ & 32 & & 32 \\
\hline Autres départements INRA & 25 & 2 & 27 \\
\hline Autres & & & 80 \\
\hline Total & 76 & 4 & 792 \\
\hline
\end{tabular}

1 Classement sur la base du premier auteur.

$21 \mathrm{er}$ auteur non INRA. 


\section{5 / Diffusion de la revue}

\section{1 / Diffusion papier}

Actuellement, la diffusion de la revue par abonnement est en moyenne de 700 exemplaires (500 payants et 200 gratuits pour un tirage habituel en 800 exemplaires), auxquels s'ajoutent une cinquantaine d'exemplaires vendus au numéro, voire davantage pour les numéros spéciaux. Ainsi, malgré un tirage supérieur (900-1000 exemplaires), ces derniers sont en effet souvent rapidement épuisés (nos Phosphore, Bien-être animal...).

Les abonnements payants se répartissent actuellement entre $70 \%$ en France et $30 \%$ à l'étranger, essentiellement en Europe ; l'Espagne, l'Italie, la Belgique et la Suisse étant les pays les plus représentés.

La répartition des abonnements français payants par secteur d'activité montre qu'environ la moitié des abonnements sont souscrits par le secteur public (30\% enseignement et $20 \%$ recherche), l'autre moitié par le secteur professionnel $(30 \%$ organismes de développement et $20 \%$ industrie).

Le nombre total d'abonnements payants diminue régulièrement avec une érosion d'environ 4\% par an. Cette diminution correspond à l'évolution globale constatée ces dernières années dans le secteur de l'édition et paraît sans lien direct avec la mise en ligne des articles.

\section{2 / Diffusion Web}

Parmi les évolutions majeures de la revue, il faut bien sûr insister sur l'ouverture de son site web dès 1996, avec la mise en ligne d'abord des seuls résumés, puis en 1997 des articles complets au format html, et enfin en 2001 des textes au format pdf, permettant ainsi le téléchargement de versions électroniques identiques aux versions papier. Les statistiques de connexion au site comportent de très nombreuses données dont l'étude est intéressante surtout en terme d'évolution. Nous retiendrons ici essentiellement deux indicateurs de l'évolution globale : le nombre de visiteurs différents et le nombre d'articles téléchargés au format pdf.

\section{a) Le nombre de visiteurs}

Le nombre de personnes visitant le site n'est bien sûr pas connu, mais il peut s'appréhender par le nombre d'ordinateurs différents s'étant connecté au cours d'une période donnée. En 1998, un an après l'ouverture du site, il était en moyenne de 700 par mois. Il a augmenté régulièrement et a atteint 38000 en moyenne chaque mois en 2007 (figure 2).

La répartition de ces ordinateurs par nom de domaine permet d'avoir une idée globale de la répartition géographique des visiteurs (sur la base du nombre global de requêtes). C'est une vue partielle car un peu plus de la moitié des noms de domaine sont génériques (.com, .net...) ou inconnus.

En 1998, 59\% des accès au site de la revue provenaient d'ordinateurs des domaines .net, .com et inconnu, 24\% de France (dont 3,2 de l'INRA) et $17 \%$ d'autres pays, essentiellement des pays européens et le Canada.

En 2007, la répartition globale a un peu changé : $51 \%$ des visites en provenance des domaines .net, .com et inconnu, 34\% de France (dont 1,6 de l'INRA) et $15 \%$ d'autres pays. Mais c'est surtout l'importance relative de chaque pays qui a évolué. Trois pays se distinguent nettement : le Maroc, la Belgique et le Canada, avec chacun plus de 150000 requêtes totales par an, ce qui correspond en tendance à plus de 10000 articles (pdf) téléchargés dans chaque pays en 2007. Viennent ensuite la Suisse,
l'Italie, le Brésil, l'Estonie, le Portugal, l'Espagne, le Mexique, les USA, l'Allemagne et les Pays-Bas avec un nombre estimé de 1000 à 5000 articles (pdf) par pays pour l'année 2007.

Cette évolution relative s'explique probablement surtout par l'augmentation des équipements informatiques et l'amélioration des caractéristiques techniques des connexions, qui nous ont permis de diffuser à l'étranger auprès d'un lectorat individuel qu'il n'était pas possible d'atteindre via les abonnements à la revue.

\section{b) Les consultations et télécharge-} ments

L'évolution de la consultation des articles (requêtes aux fichiers html) a été la plus importante de 1999 à 2003 puisqu'elle a été multipliée par 7 au cours de cette période, passant de 10000 à 70000 par mois. Elle a encore progressé de 2003 à 2007 pour atteindre désormais 100000 consultations en moyenne par mois.

Depuis la mise en ligne des articles au format pdf, en accès gratuit, le site totalise plus de $\mathbf{2}$ millions de téléchargements en 7 ans. L'augmentation du nombre de téléchargements par mois a été particulièrement forte $(+273 \%)$ entre 2001 et 2003 , puis l'évolution s'est stabilisée autour de $+20 \%$ par an jusqu'en 2005 où l'on dépasse 30000 articles téléchargés chaque mois. A partir de 2006, l'augmentation plus soutenue (figure 3)
Figure 2. Nombre de visiteurs différents par mois.

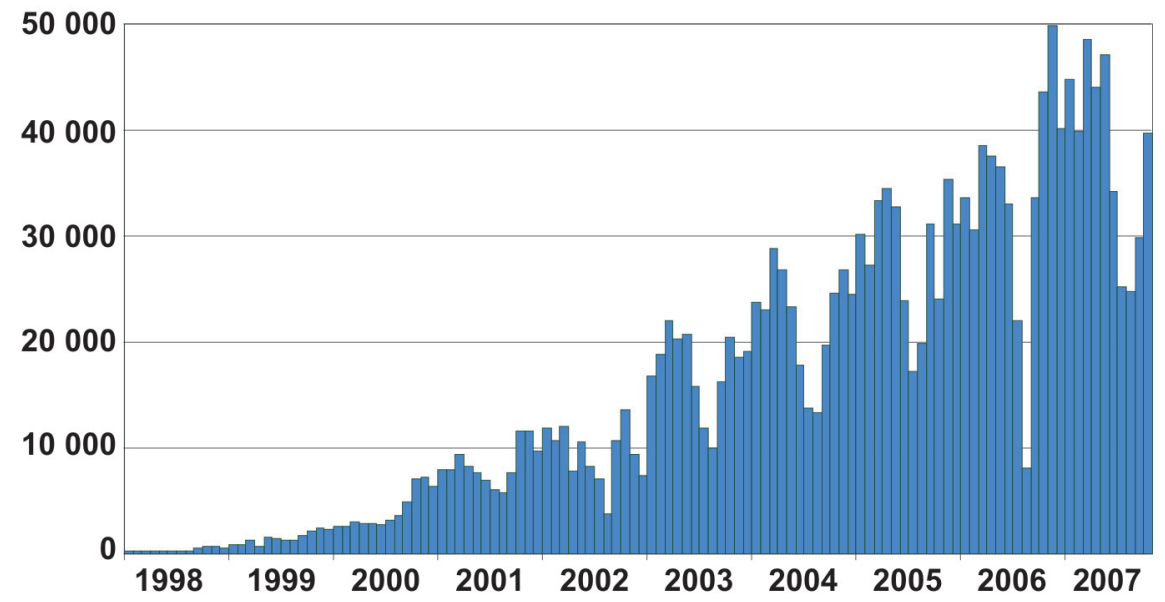


s'explique en partie par des modifications de présentation des articles sur le site, mais aussi par le nombre de téléchargements particulièrement élevé pour certains articles (ex. plus de 2000 téléchargements d'un même article en un mois).

Si les articles les plus récents bénéficient généralement d'un plus grand nombre de téléchargements l'année où ils sont publiés, les articles plus anciens intéressent aussi les lecteurs. Afin de mettre à leur disposition tous les articles publiés, les anciens numéros de la revue (1988-1995) ont été numérisés et la quasi-totalité 1 des articles parus est désormais accessible gratuitement sur le nouveau site web mis en place en février 2008 à l'occasion du 20 eme anniversaire de la revue.
Figure 3. Nombre d'articles (pdf) téléchargés par mois.

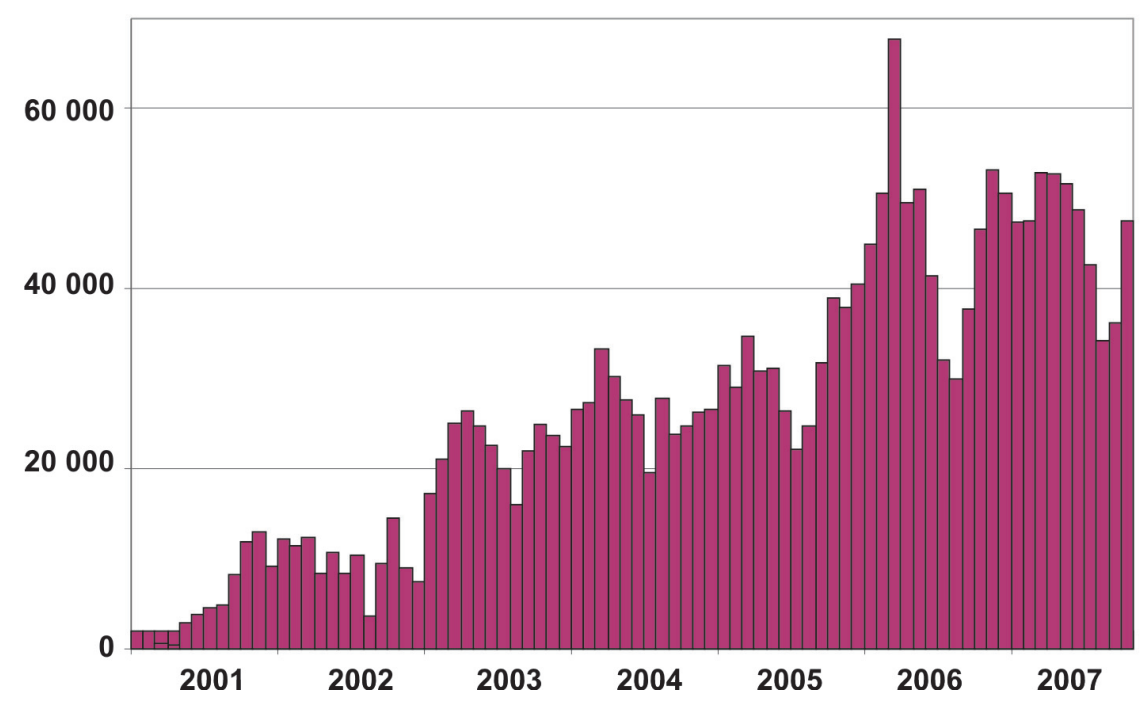

\footnotetext{
${ }^{1}$ Les numéros spéciaux et hors série sont mis en ligne sur le site web de la revue après épuisement de la version papier.
} 
